(3) There exists a p-ordered sequence of homogeneous generators of $I$ $u_{1}, \ldots, u_{p}, \ldots, u_{n}, h\left(u_{i}\right)=h_{i}$, such that the Tate resolution $X$ of $A$ is equal to

$X=F_{2} X=A\left\langle T_{1}, \ldots, T_{n}, d T_{i}=u_{i}\right\rangle\left\langle S_{p+1}, \ldots, S_{n}, d S_{j}=u_{j}^{h j-1} T_{j}\right\rangle$

with $\partial\left(T_{i}\right)=\partial\left(u_{i}\right), w\left(T_{i}\right)=1, \partial\left(S_{j}\right)=h_{j} \partial\left(u_{j}\right), w\left(S_{j}\right)=2$.

(4) There exists a $p$-ordered sequence $u_{1}, \ldots, u_{p}, \ldots, u_{n}$ of homogeneous generators of the ideal $I, h\left(u_{i}\right)=h_{i}$, such that

$$
H_{1}(\boldsymbol{B})=H_{2}(\boldsymbol{B})=0
$$

where $\boldsymbol{B}=A\left\langle T_{1}, \ldots, T_{n}, d T_{i}=u_{i}\right\rangle\left\langle S_{p+1}, \ldots, S_{n}, d S_{j}=u_{j}^{h-1} T_{j}\right\rangle$.

Proof. The equivalence of (1) and (2) is contained in Proposition 1.14. Implication $(2) \Rightarrow 3$ ) follows from Oorollary 2.8 and $(3) \Rightarrow(4)$ is obvious. Finally, implication $(4) \Rightarrow(3)$ holds in virtue of Proposition 2.6.

2.11. Remark. We do not know if the above theorem is true for graded $R$-algebras which are not finitely generated.

The following example shows that not every graded $R$-algebra $A$ with $X=F_{2} X$ is $h$-regular, i.e. satisfies one of the equivalent conditions from Theorem 2.10.

2.12. EXAMPLE. Let $k$ be a field and let $A=k[X, Y] /(X Y)$, where $\partial(X)=\partial(Y)=2$. Since $X Y$ is a non-zero divisor in $k[X, Y]$, we have $X=F_{2} X$ by [2], IV, $\S 2$ Theorem 1. At the same time one can easily prove that $A$ is not an $h$-regular graded $k$-algebra.

\title{
References
}

[1] T. Józefiak, Tate resolution for commutative graded algebras over a local ring, Fund. Math. 74 (1972), pp. 209-231.

[2] - Thesis, Toruń 1969.

[3] - A homological characterization of graded complete intersections, to appear.

[4] A. Tyc, On $h$-regular local Noetherian rings, to appear.

INSTITUTE OF MATHEMATICS, POLISH ACADEMY OF SCIENCES

Reģu par la Rédaction le 8. 1. 1973

\section{Absolute retracts as factors of normed linear spaces}

by

\section{H. Toruńczyk (Warszawa)}

Abstract. It is shown that if $X$ is a (complete) $A R(\mathfrak{M})$-space, then, for a suitable (complete) normed linear space $E, X \times E$ and $E$ are homeomorphic. This implies that $l_{2}$-manifolds are characterized as separable, complete, $l_{2}$-stable $\operatorname{ANR}(\mathfrak{M})$ 's.

In this paper we deal with products of absolute retracts and normed linear spaces. We shall show that any absolute retract is a factor of a normed linear space, i.e. if $X \in \mathrm{AR}(\mathfrak{M})$, then there is a normed space $E$ such that $X \times E$ is homeomorphic to $E$. We also show that normed spaces $E$ of a more special type (e.g. all infinite-dimensional Hilbert spaces) have the property that each retract of $E$ is also a factor of $E$.

The paper is a sequel to [28] and we shall use some terms and notation of [28]. In particular, we shall say that a retraction $r$ of a metric space $(Y, d)$ is regular, if $r$ is continuous and

(*) for every $\varepsilon>0$ the set $\{y \in Y: d(r(y), y) \geqslant \varepsilon\}$ is of positive $d$-distance from $r(Y)$.

The main result of [28] was:

THEOREM 0 . Let $r$ be a regular retraction of a normed lnnear space $(E,\|\|)$ and let $X=r(E)$. Then $X \times \sum_{l_{1}} E \cong \sum_{l_{1}} E$, and if, moreover, $X$ is complete in the norm \|\| , then also $X \times \prod_{l_{1}} E \cong \prod_{l_{1}} E$. Here, $\prod_{l_{1}} E$ $=\left\{\left(t_{i}\right) \in \mathbb{E}^{\infty}: \sum\left\|t_{i}\right\|<\infty\right\}$ and $\sum_{l_{1}} E=\left\{\left(t_{i}\right) \in \mathbb{E}^{\infty}: t_{i}=0\right.$ for almost all $\left.i\right\}$, both spaces being equipped with the norm $\left\|\left(t_{i}\right)\right\|\left\|=\sum_{i \geqslant 1}\right\| t_{i} \|$.

To apply this theorem, we examine here regular retractions more accurately. Unexpectedly enough, it appears (see Section 2) that on any absolute retract $\bar{X}$ there is an admissible metric $\varrho$ with the property that every closed isometric embedding of $(X, \varrho)$ into a metric space $\bar{Y}$ maps $\bar{X}$ 
onto a regular retract of $Y$. Combining this with the Arens-Eells Embedding Theorem, we obtain in Section 3 the above-mentioned results concerning products of absolute retracts and normed spaces, and we use them next in discussing the following subjects: (i) products of ANR's and normed spaces and the identifying of $l_{2}$-manifolds (Section 4 ); (ii) products and factors of simplicial complexes with metric topology (Section 5), and (iii) tubular neighbourhoods of $Z$-embeddings of ANR's (Section 6). Section 1 is of preliminary character; for convenience in references we include in it the results of [5], [20] and [27] which we need in this paper.

The author would like to thank Cz. Bessaga and P. Mine for valuable conversations during the preparation of this paper.

1. Preliminaries. Throughout the paper we shall denote by $R$ the set of real numbers and by $N$ the set of positive integers. The elements of the extended reals $[-\infty, \infty]$, as well as the $[-\infty, \infty]$-valued functions, will be denoted by the Greek letters $\varepsilon, \delta, \lambda, \mu$, while positive integers will be denoted by $i, j, k, n$. The closed intervals of both $[-\infty, \infty]$ and $N$ will be denoted by $[\varepsilon, \delta]$; thus, $\{\lambda: \lambda \in[\varepsilon, \delta]\}$ is the "real" interval, while by $\{i: i \in[\varepsilon, \delta]\}$ we mean the intersection of this interval with $N$. We assume $\infty+\infty=\infty$,

If not stated otherwise, by "retraction" we mean "continuous retraction". We shall say " $r$ is a regular retraction of $Y$ " instead of " $r$ is a regular retraction of $(Y, d)$ " if the metric $d$ is clear from the context. The notation and definitions concerning Absolute Retracts and Absolute Neighbourhood Retracts are those of [7].

Given a metrizable space $X$ we denote by $\operatorname{Metr}(X)$ the set of all metrics on $X$ which induce the topology of $X$; elements of $M$ Metr $(X)$ will be called admissible metrics for $X$. If $\varrho \in \operatorname{Metr}(X)$ and $A \subset X$, then for $x \in X$ we let $\operatorname{dist}_{e}(x, A)=\inf \{\varrho(x, a): a \in A\}$. By dens $(X)$ we mean the least cardinality of dense subsets of $X$, card $(A)$ denotes the cardinality of a set $A$, and we shall write $\left(X, A_{1}, \ldots, A_{n}\right) \cong\left(X, B_{1}, \ldots, B_{n}\right)$ to indicate that there is a homeomorphism $f: X \stackrel{\text { onto }}{\longrightarrow} X$ with $f\left(A_{i}\right)=B_{i}$ for $i \in[1, n] . O(T, X)$ denotes the space of all continuous maps from $T$ to $X$.

Let $(\boldsymbol{E},\|\|)$ be a normed linear space. By $\mathbb{E}^{\infty}$ we denote the countable Cartesian power of $E$, considered in the product topology, and we let $\sum E=\left\{\left(t_{i}\right) \in E^{\infty}: t_{i}=0\right.$ for almost all $\left.i\right\}$ be the topological subspace of $E^{\infty}$ (not to be confused with the space $\sum_{l_{1}} E$ defined in the formulation of Theorem 0 ). The convex hull of a set $A \subset B$ will be denoted by conv $A$.

For the sake of convenience let us introduce the following notation: on $A$;

$\mathbb{H}_{1}(A)=l_{2}(A)$, the Hilbert space of square-summable real functions
$A$;

$$
E_{2}(A)=l_{2}(A) \times \sum R
$$

$E_{3}(A)=l_{2}^{f}(A) \stackrel{\text { df }}{=}\left\{\lambda \in l_{2}(A): \lambda(a)=0\right.$ for almost all $\left.a \in A\right\}$;

$E_{4}(A)=l_{2}^{f}(A) \times Q$, where $Q=[-1,1]^{\infty}$ is the Hilbert cube.

To those spaces there correspond some classes of metric spaces, which we shall denote as follows:

$\mathfrak{M}_{\mathrm{j}}=$ the class of complete-metrizable spaces;

$\mathfrak{M}_{2}=$ the class of metric spaces which are countable unions of elosed, complete-metrizable sets;

$\mathfrak{M}_{3}=$ the class of metric spaces which are countable unions of locally compact, locally finite-dimensional sets;

$\mathfrak{M}_{4}=$ the class of metric spaces which are countable unions of locally compact sets.

The spaces $l_{2}(N)$ and $l_{2}^{f}(N)$ will also be denoted by $l_{2}$ and $l_{2}^{f}$ respectively. Below we list some results describing the properties of the spaces $\mathbb{H}_{i}(A)$, $i \in[1,4]$ :

1.1. Let $i \in[1,4]$ and let $A$ be an arbitrary set. Then a space $X$ with dens $(X) \leqslant \operatorname{cord}(A)$ admits a olosed embedding into $\mathbb{H}_{i}(A)$ if and onty if $X \in \mathfrak{M i}_{i}$.

Proof. The assertion is well known for $i=1$ (see [20], proof of Corollary 2.4 or [6], p. 606); for the proof in the case $i \in[2,4]$ see [27], $\S 7$.

1.2. For any infinite set $A$ we have:

(a) $\sum R \cong l_{2}^{f}$ and $\mathbb{H}_{2}(A) \cong l_{2}(A) \times l_{2}^{f}$;

(b) $l_{2}^{f} \times Q \cong \mathbb{H}_{\sigma}$ and (hence) $\mathbb{E}_{4}(A) \cong l_{2}^{f}(A) \times E_{\sigma}$, where $\mathbb{E}_{\sigma}=\left\{\lambda \in l_{2}\right.$ : $\left.\sum i^{2} \lambda(i)^{2}<\infty\right\}$ is the subspace of $l_{2}$.

In particular, each of the spaces $\mathbb{E}_{i}(A), i \in[1,4]$, is homeomorphio to a pre-Hilbert space.

For a proof see [5], §5, and [27], §7.

1.3. Let $X$ be a space with $X \times E_{2}(A) \cong E_{2}(A)$ and let $i \epsilon\{3,4\}$. If $X \in \mathfrak{M}_{i}$ then $X \times \mathbb{H}_{i}(A) \cong \mathbb{E}_{i}(A)$.

Proof. Assume first $i=3$. It follows from [27] that $X \times l_{2}^{f}(A) \times l_{2}^{f}$ and $l_{2}^{f}(A) \times l_{2}^{f}$ are $\nVdash$-absorbing sets in $X \times l_{2}(A) \times l_{2}^{f}$ and $l_{2}(A) \times l_{2}^{\prime}$ respectively, where $\pi$ denotes the family of all locally compact finite-dimenssional subsets of the space in question. Since $l_{2}(A) \times l_{2}^{f} \cong E_{2}(A)$ (see 1.2), it follows from our assumption and from the general properties of absorbing sets that $X \times l_{2}^{f}(A) \times l_{2}^{f} \cong l_{2}^{f}(A) \times l_{2}^{f}$.

In the case $i=4$ the proof is similar (replace $l_{2}^{f}(A) \times l_{2}^{f}$ by $E_{4}(A)$ and $\pi$ by the family of all locally compact sets).

1.4. Let $(E,\|\|)$ be a normed linear space and let $A$ be a set of cardinality dens $(E)$. 
(a) If $E$ is a Banach space then there exsists a space $F$ such that $E \times F \cong l_{2}(A)$;

(b) If $E$ is a countable union of its closed subsets, each being complete in the norm \|\| , then $E \times E_{2}(A) \cong E_{2}(A)$.

Proof. Part (a) is shown in [20], pp. 28-29 (cf. [4], p. 266) and part (b) in [27], §7.

2. Regular retractions and regular metrics. Let $X$ be an $\operatorname{AR}(\mathfrak{M})$-space. In this section we shall be concerned with the question whether $X$ can be embedded in a normed linear space as its regular retiract. Let us make some introductory remarks. Assume for simplicity that $X$ is a closed subset of a normed linear space $(E,\|\|)$. By the definition of $\operatorname{AR}(\mathfrak{M})$ spaces, there is in this case a retraction $r: E \stackrel{\text { onto }}{\rightarrow} X$; unfortunately, $r$ need not be regular in any translation-invariant metric of $E$. For example, if $E_{0}$ denotes the Euclidean plane and $X_{0}$ is the set

$$
\{1\} \times[0,1] \cup\left\{\left(x_{1}, 0\right): x_{1} \geqslant 1\right\} \cup\left\{\left(x_{1}, 1 / x_{1}\right): x_{1} \geqslant 1\right\},
$$

then it is easy to observe that there is no regular retraction of $E_{0}$ onto $X_{0}$. Obviously $X_{0}$ is homeomorphic to $X_{1}=\left\{\left(x_{1}, 0\right): x_{1} \in R\right\}$, which is a regular retract of $E_{0}$. To get the required re-embedding in this special case we had to "push apart" some "parts" of $X_{0}$; however, it is not clear how these parts should be defined in the general situation $\left({ }^{1}\right)$. Are they, for instance, simply neighbourhoods of distinct points of the remainder of a properly defined completion of $X$ ? It is so for $(E, X)=\left(E_{0}, X_{0}\right)$ but when drawing other examples it appears that one should rather depend on the interrelation of a fixed retraction $r: E \stackrel{\text { onto }}{\longrightarrow} X$ and the affine stiructure of $E$ : e.g., in any of the simplest examples one has to "push apart" any two sequences $\left(a_{n}\right)$ and $\left(b_{n}\right)$ of points of $X$ for which we have both $\inf \left\|a_{n}-b_{n}\right\|=0$ and $\inf \left\{\operatorname{diam}_{\|\|} r\left(\left[a_{n}, b_{n}\right]\right): n \in N\right\}>0 \quad\left(\left[a_{n}, b_{n}\right]\right.$ denotes $n$

$\left.\left\{\lambda a_{n}+(1-\lambda) b_{n}: \lambda \in[0,1]\right\}\right)$. Extending this idea, we shall obtain the required re-embedding in two steps: we construct first a metric $\varrho$ on $X$ such that for no sequence $\left(A_{n}\right)$ of subsets of $X$ we have $\inf \left\{\operatorname{diam}_{0} A_{n}\right.$ : $n \in N\}=0$ while $\inf \left\{\operatorname{diam}_{e} r\left(\operatorname{conv} A_{n}\right): n \in N\right\}>0$, and we then show that any closed isometric embedding of $(X, \varrho)$ into a normed space $(F,||||||)$ maps $X$ onto a regular retract of $F$.

The detailed proofs run as follows:

2.1. Proposition. Let $r: K \stackrel{\text { onto }}{\longrightarrow} X$ be a retraction of a convex subset $K$ of a locally convex linear metric space. Then, for every $\varrho_{0} \in \operatorname{Metr}(X)$, there (I) We say that a map $h:(X, \varrho) \rightarrow(Y, d)$ pushes the sets $A, B \subset X$ apart, if
dist $_{e}(A, B)=0$ while dist dis $_{d}(h(A), h(B))>0$.

is a $\varrho \in \operatorname{Metr}(X)$ and a funation $\varepsilon:[0, \infty] \rightarrow[0, \infty]$ such that: (1) $\lim _{\delta \rightarrow 0} \varepsilon(\delta)$ $=0$, (2) for every $A \subset X$ the $\varrho$-diameter of $r$ (conv $A)$ does not exceed $\varepsilon\left(\operatorname{diam}_{\varrho} A\right)$, and $(3) \varrho \geqslant \varrho_{0}$.

The $\varrho-\varrho_{0}$ will be obtained as a sum of a countable number of metries, each built inductively on the basis of the following

SUBLEMma. Let $K, X$ and $r$ be as above and let $\varrho_{i} \in \operatorname{Metr}(X)$ be fixed Then there exists $a \varrho_{i+1} \in \operatorname{Metr}(X)$ such that

(*i) For any $n \in N$ and $A \subset X$ with $\operatorname{diam}_{e i+1} A \leqslant 2^{-n}$, we have

$$
\operatorname{diam}_{\mathrm{er}} r(\operatorname{conv} A) \leqslant n^{-1} 2^{-n} \text {. }
$$

Proof. Given $n \in N$, let $\mathcal{U}_{n}$ be a cover of $X$ consisting of (relatively) open sets which are so small that $\operatorname{diam}_{e i} r(\operatorname{conv} U) \leqslant n^{-1} 2^{-n}$ for all $U \epsilon \mathcal{U}_{n}$. By a lemma of E. Michael, there are metrics $d_{n}, n \in N$, such that every set of $\boldsymbol{d}_{n}$-diameter less than 1 is contained in a member of $\mathcal{U}_{n}$ ([23], p. 165). We let

$$
\varrho_{i+1}\left(x_{1}, x_{2}\right)=\sum_{n=1}^{\infty} \min \left(d_{n}\left(x_{1}, x_{2}\right), 2^{-n+1}\right), \quad x_{1}, x_{2} \in X .
$$

Proof of Proposition 2.1. Starting from the metric $\varrho_{0}$, construct inductively a sequence $\varrho_{1}, \varrho_{2}, \ldots$ of admissible metrics on $\ddot{X}$ satisfying for every $i \geqslant 0$ condition (*i), and define $\varrho \in \operatorname{Metr}(X)$ by the formula

$$
\varrho\left(x_{1}, x_{2}\right)=\varrho_{0}\left(x_{1}, x_{2}\right)+\sum_{i=1}^{\infty} \min \left(\varrho_{i}\left(x_{1}, x_{2}\right), 2^{-i+1}\right), \quad x_{1}, x_{2} \in X .
$$

If $A \subset X$ is a set with $\operatorname{diam}_{e} A \leqslant 2^{-n}$, then for all $i \epsilon[1, n]$ we have $\operatorname{diam}_{e_{i}} A \leqslant 2^{-n}$. This gives $\operatorname{diam}_{e_{i}} r(\operatorname{con} \nabla A) \leqslant n^{-1} 2^{-n}$ for $i \epsilon[0, n-1]$ and, consequently, diam $r(\operatorname{con} \nabla A) \leqslant n \cdot n^{-1} \cdot 2^{-n}+\sum_{i \geq n} 2^{-i+1}=5 \cdot 2^{-n}$. Thus, the metric $\varrho$ and the function $\varepsilon$ defined by

$$
\varepsilon(\delta)=10 \delta \text { if } \delta \epsilon[0,1 / 2] \text { and } \quad \varepsilon(\delta)=-\infty \text { if } \delta>1 / 2
$$

satisfy the required conditions.

By a uniform embedding of a metric space $(X, \varrho)$ into another one $(Y, d)$ we shall mean here any embedding $h: X \rightarrow Y$ such that both $h$ and $h^{-1}$ are uniformly continuous when considered as maps between the metric spaces $(X, \varrho)$ and $(h(X), d)$.

2.2. Propostrion. Let $K$ be a convex set in a linear metric space, let $r: K \stackrel{\text { onto }}{\longrightarrow} X \subset K$ be a retraction, and let $\varrho \in \operatorname{Metr}(X)$ and $a$ function $\varepsilon$ : $(0, \infty] \rightarrow(0, \infty]$ satisfy conditions (1) and (2) of Theorem 2.1. Then, for 
every closed uniform embedding $h$ of $(X, \varrho)$ into a metric space $(X, d)$, there is a regular retraction of $(Y, d)$ onto $h(X)$.

Proof. Let $\left(\left(\lambda_{U}, z_{U}\right)\right)_{U \epsilon}$ be a Dugundji system for $(Y, h(X), d)$, i.e. we assume that $\left(\lambda_{U}\right)_{U \in U} U$ is a (continuous and locally finite) partition of unity on $\bar{Y} h(X)$ and, for every $U \epsilon \mathcal{U}, z_{U}$ is a point of $h(X)$ such that

(D) if $y \in \bar{Y}$ satisfies $\lambda_{U}(y) \neq 0$, then $d\left(y, z_{U}\right) \leqslant 4 \operatorname{dist}_{d}(y, h(X))$

(for the construction of systems of the required type see [8], p. 188).

We define a retraction $q: Y \stackrel{\text { onto }}{\longrightarrow} h(X)$ by the formula

$$
q(y)=\left\{\begin{array}{lll}
y & \text { if } & y \in h(X), \\
h r\left(\sum_{U \in \mathcal{U}} \lambda_{U}(y) \cdot h^{-1}\left(z_{U}\right)\right) & \text { if } & y \in X \backslash h(X) .
\end{array}\right.
$$

Obviously, $q$ is continuous on the open set $\bar{X} h(X)$. Thus, the proof will be completed if we show that $q$ satisfies condition (*) (for $q$ will then also be a continuous map).

For $n=1,2$, let $\varepsilon_{n}:(0, \infty] \rightarrow(0, \infty]$ be a function such that $\lim _{\delta \rightarrow 0} \varepsilon_{n}(\delta)$ $=0$ and

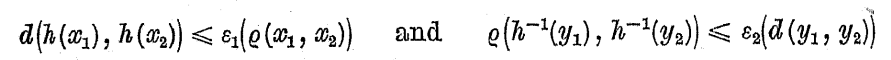

for all $x_{1}, x_{2} \in X$ and $y_{1}, y_{2} \in Y$. Without loss of generality assume that $\varepsilon_{1}, \varepsilon_{2}$ and $\varepsilon$ are non-decreasing functions. Fix for a moment $y \in \bar{Y}$, let $\mathcal{U}_{0}$ denote the finite set $\left\{U \in \mathcal{U}: \lambda_{U}(y) \neq 0\right\}$, and choose $V \in \mathcal{U}_{0}$. Setting $\delta=\delta(y)=\operatorname{dist}_{d}(y, h(X))$, we have $d\left(y, z_{U}\right) \leqslant 4 \delta$ for all $U \in \mathcal{U}_{0}$, whence $\operatorname{diam}_{d}\left\{z_{U}: U \in \mathcal{U}_{0}\right\} \leqslant 8 \delta$ and $\operatorname{diam}_{e}\left\{h^{-1}\left(z_{U}\right): U \in \mathcal{U}_{0}\right\} \leqslant \varepsilon_{2}(8 \delta)$. This gives $\operatorname{diam}_{e} r\left(\operatorname{con} \nabla\left\{h^{-1}\left(z_{U}\right): U \in \mathcal{U}_{0}\right\}\right) \leqslant \varepsilon \circ \varepsilon_{2}(8 \delta)$ and, in particular,

$$
\varrho\left(h_{-}^{-1} q(y), h^{-1}\left(z_{V}\right)\right) \leqslant \varepsilon \circ \varepsilon_{2}(8 \delta) \text {. }
$$

Letting $\varepsilon_{0}(\mu)=4 \mu+\varepsilon_{1} \circ \varepsilon \circ \varepsilon_{2}(8 \mu)$, we get $d\left(q(y), z_{V}\right) \leqslant \varepsilon_{1} \circ \varepsilon \circ \varepsilon_{2}(8 \delta)$ and $d(q(y), y) \leqslant \varepsilon_{0}(\delta)=\varepsilon_{0}(\delta(y))$. Since this inequality holds true for all $y \in Y$ and since $\lim _{\mu \rightarrow 0} \varepsilon_{0}(\mu)=0$, the assertion is proved.

Let us say that a metric $\varrho$ defined on an $\operatorname{AR}(\mathfrak{M})$-space $X$ is a regular metric for $X$ if $\varrho \in \operatorname{Metr}(X)$ and, given any closed uniform embedding of $(X, \varrho)$ into a metric space $Y$, the image of $X$ is a regular retract of $X$. Using the fact that every metric space admits a closed embedding into a convex subset of a normed linear space (Kuratowski, Eilenberg and Wojdysławski), we infer from Propositions 2.1 and 2.2:

2.3. THEOREM. If $X$ is an $\operatorname{AR}(\mathfrak{M})$-space, then for every $\varrho_{0} \in \operatorname{Metr}(X)$ there exists a regular metric $\varrho$ for $X$ such that $\varrho \geqslant \varrho_{0}$.
The applications of 2.3 discussed in the subsequent sections depend on the following theorem of R. Arens and J. Eells:

( $\mathrm{AE})$ Every metric space $(X, \varrho)$ admits an isometric embedding $h$ into a Banach space, such that the set $h(X)$ is both linearly independent and closed in its linear span.

This theorem, which generalizes earlier results of Kuratowski, Kunugui and Eilenberg-Wojdysławski, was established in [3]; for some shorter proofs see [24] or [30]. Obviously, (AE) and 2.3 imply a positive answer to the question stated at the beginning of this section.

3. Absolute retracts as factors of normed spaces. Theorems 0 and 2.3 combined with the theorem of Arens and Eells yield:

3.1. THEOREM. Given $X \in \mathrm{AR}(\mathfrak{M})$ there is a normed linear space $E$ such that $X \times E \cong \mathbb{E}$ and $\operatorname{dens}(E)=\operatorname{dens}(X)$.

Unfortunately, 3.1 does not give any information on the topological type of the normed space whose factor is $X$. The remainder of the section will be devoted to obtaining some results in this direction, at least for absolute retracts belonging to one of the classes $\mathfrak{M}_{i}, . i \in[1,4]$, discussed in Section 1.

3.2. THEOREMr. Let $i \in[1,4]$. If $X$ is an $\operatorname{AR}(\mathfrak{M})$-space with $X \in \mathfrak{M}_{i}$, then $X \times E_{i}(A) \cong E_{i}(\mathcal{A})$, where $A$ is a set of cardinality dens $(X)$.

Proof. We shall consider separately the cases $i=1, i=2$ and $i \in\{3,4\}$.

(a) $i=1$. Let $\varrho_{0} \in \operatorname{Metr}(X)$ be a complete metric on $X$, let $\varrho \in \operatorname{Metr}(X)$ be a regular metric with $\varrho \geqslant \varrho_{0}$ and let $h: X \rightarrow L$ be an isometric embedding of $(X, \varrho)$ into a Banach space $L$ of density character equal to that of $X$. Then $\varrho$ is a complete metric, and therefore $h$ is a closed embedding. By Theorem 0 we get $X \times E \cong E$, where $E$ denotes the Banach space $\prod_{l_{1}} L$. Thus $X \times E \times F \cong E \times F$ for every space $F$, and $X \times l_{2}(A) \cong l_{2}(A)$ by 1.4 (a).

(b) $i=2$. By 1.1 and 1.2 (a) $w \theta$ can consider $X$ as a closed subset of $l_{2}^{t} \oplus l_{2}(A)$. Let \|\| be the norm of $l_{2}^{t} \oplus l_{2}(A)$ and let $\varrho_{0}$ denote the metric induced by \|\| on $X$; further let $\varrho$ be a regular metric for $X$ such that $\varrho \geqslant \varrho_{0}$, and let $h$ be an isometric embedding of $(X, \varrho)$ into a Banach space $I$ with dens $(F)=\operatorname{dens}(X)$. It is easy to see that

$$
x \rightarrow(h(x), x) \in F \oplus l_{2}^{f} \oplus l_{2}(A)
$$

is a closed uniform embedding of $(X, \varrho)$ into the normed space $E=F \oplus l_{2}^{f} \oplus l_{2}(A)$. Applying Theorem 0 , we get $X \times \sum_{l_{1}} E \cong \sum_{l_{1}} E$, which

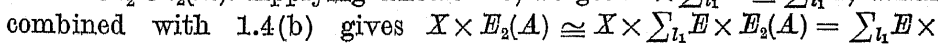
$\times \mathbb{H}_{2}(A) \cong \mathbb{H}_{2}(A)$. 
(c) $i \epsilon\{3,4\}$. We have $\mathfrak{M}_{i} \subset \mathfrak{M}_{2}$ and therefore $X \times \mathbb{H}_{2}(A) \cong \mathbb{H}_{2}(A)$ by the case (b) above. Thus the assertion follows $1.3\left(^{2}\right)$

For separable spaces the formulation of the case $i=4$ of Theorem 3.2 can be simplified:

3.3. CoRoliarY. If $X \in \mathrm{AR}(\mathfrak{M})$ is a countable union of compact sets, then $X \times \mathbb{E}_{\sigma} \cong \mathbb{E}_{\sigma}$, where $\mathbb{E}_{\sigma}=\left\{\lambda \in l_{2}: \sum_{i \geq 1} i^{2} \lambda(i)^{2}<\infty\right\}$ is the subspace of $l_{2}$.

Proof. By $1.2(\mathrm{~b})$ the space $Q \times l_{2}^{f}$ is homeomorphic to $D_{\sigma}$.

Theorem 3.2 can be equivalently stated in the following form

3.4. CoRoLiARY. Let $E$ be a space of the form $E_{i}(A)$, where $i \in[1,4]$

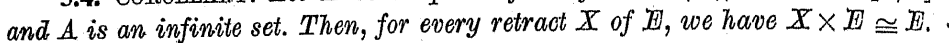

Question: Does every linear metric space $\mathbb{E}$ have the property that each retract of $E$ is a factor of both $E^{\infty}$ and $\sum E$ ?

4. Identifying of manifolds. By a cone over a metric space $X$ we mean the topological space $(C X, \mathfrak{C})=(X \times(0,1] \cup\{0\}, \mathfrak{C})$, where $\mathscr{C}$ is the topology generated by open subsets of $X \times(0,1]$ and sets $\{0\} \cup$ $\cup(0,1 / n) \times X, n \in \mathbb{N}$.

4.1. LEMara. If $E$ is a linear metric space such that either $E$ is locally convex or $E \cong E^{\infty}$ or $E \cong \sum E$, then $C E$ is homeomorphic to a retract of $E \times R$.

Proof. By results of Henderson ([11], [12, p. 322]), the open cone $O E \backslash E \times\{1\}$ is homeomorphic to $E \times R$. Since $C E$ can be embedded as a retract of $C E \backslash E \times\{1\}$, the result follows:

The following results correspond to 3.1 and 3.2 .

4.2. THEOREM. If $X$ is an $\operatorname{ANR}(\mathfrak{M})$-space, then there exists a normed linear space $E$ such that $\operatorname{dens}(E)=\operatorname{dens}(X)$ and $X \times \mathbb{E}$ is homeomorphic to an open set $U \subset \mathbb{E}$ with $(E, E \backslash U) \cong(C X \times E,\{0\} \times E)$.

Proof. By (AE), one can consider $X$ as a neighbourhood retract of a normed linear space $F^{\text {with }}$ dens $(F)=\operatorname{dens}(X)$. As is easy to see, $O X$ is a retract of $C F$ (cf. [16], proof on p. 43) and therefore, by Lemma 4.1, $O X$ can be treated as a retract of $F \times R$. Thus there is a normed linear space $E_{1}$ such that dens $\left(E_{1}\right)=\operatorname{dens}(X)$ and $C X \times \mathbb{E}_{1} \cong \mathbb{E}_{1}$. We set $E$

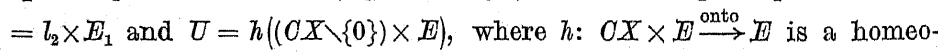
morphism. Then $U \cong X \times(0,1] \times \mathbb{E} \cong X \times\left((0,1] \times l_{2}\right) \times \mathbb{E}_{1}$; since $(0,1] \times$ $\times l_{2} \cong l_{2}$ (this result belongs to $\mathrm{V}$. L. Klee and follows also directly from Theorem 3.2), we have $U \cong X \times l_{2} \times E_{1} \cong X \times \mathbb{E}$, which completes the proof.

(2) In the separable case the use of 1.3 can be avoided. (Follow the proof of (b) and use results of Klee [18, p. 190] and of Bessaga-Pelczyński [5, pp. 176-178], cf. $[28$, p. 82$]$.)
4.3. THEOREM. Let $i \in[1,4]$, let $X \in \mathfrak{M}_{i}$, and let $A$ be a set of cardinality dens $(X)$. If $X \in \operatorname{ANR}(\mathfrak{M})$, then $X \times \mathbb{E}_{i}(A)$ is homeomorphic to an open set $U \subset \mathbb{E}_{i}(A)$ such that $\left(\mathbb{E}_{i}(A), \mathbb{E}_{i}(A) \backslash U\right) \cong\left(C X \times E_{i}(A),\{0\} \times E_{i}(A)\right)$.

The proof is analogous to that of Theorem 4.2.

Theorem 4.3 implies the following version of a special case of Henderson's [12] Open Embedding Theorem:

4.4. Corollary. Let $E$ be as in 4.3 and let $X$ be a paracompact connected manifold modelled on $E$. Then $X$ is homeomorphic to an open set $U \subset \mathbb{U}$ such that $(E, \mathbb{E} \backslash U) \cong(O X \times \mathbb{E},\{0\} \times \mathbb{E})$.

Proof. By [15], Theorem 6, $X$ admits a closed embedding into $E$, and by results of Anderson and Schori [2], [26] the spaces $X \times X$ and $X$ are homeomorphic $\left({ }^{3}\right)$. Using this, we obtain the assertion from 4.3 and a result of $[25], p .3$, which states that $X \in \operatorname{ANR}(\mathfrak{M})$.

Also, we obtain the following characterization of certain infinitedimensional manifolds:

4.5. Propostition. Let $X$ be a metric space, let $A$ be a set of cardinality dens $(X)$ and let $i \in[1,4]$. Then, $X$ is an $E_{i}(A)$-manifold if and only if $X$ is $\mathbb{E}_{i}(A)$-stable (i.e. $\left.X \times \mathbb{E}_{i}(A) \cong \bar{X}\right)$ and $X \in \mathfrak{M}_{i} \cap \operatorname{ANR}(\mathfrak{M})$.

Proof. 1.1 and the proof of 4.4 combine to show that every $E_{i}(A)$-manifold is an $E_{i}(A)$-stable space belonging to $\mathfrak{M}_{i} \cap \operatorname{ANR}(\mathfrak{M})$. The reverse implication follows from 4.4 .

Below, two applications of Proposition 4.6 to identifying $l_{2}$-manifolds are given; they depend on "stability theorems" for spaces of continuous mappings, due to R. Geoghegan and J. Keesling.

4.6. Corollary. Let $X$ be a separable complete ANR $(\mathfrak{M})$-space, $X_{1}, \ldots, X_{n}$ its ANR (M)-subsets of type $G_{\delta}, T$ a compact space and $T_{1}, \ldots, T_{n} \subset T$ disjoint closed sets. If $U$ is a cone-patch (in sense of [10]) for $T$ such that $U \subset T_{1}$ or $U \cap\left(T_{1} \cup \ldots \cup T_{n}\right)=\emptyset$ then the space $\{f \epsilon O(T, X)$ : $f$ is non-constant on $U$ and $f\left(T_{i}\right) \subset X_{i}$ for $\left.i \in[1, n]\right\}$ forms an $l_{r_{2}}$-manifold when considered in the compact-open topology.

Proof. By results of Geoghegan (see proofs on pp. 168-169, 171 and $174-175$ in [10]) the space in question is $l_{2}$-stable. Since it is also an open subset of $\bar{Y}=\left\{f \in C(T, X): f\left(T_{i}\right) \subset X_{i}\right.$ for $\left.i \epsilon[1, n]\right\}$, the result follows from 4.5 and the fact that $Y$ is a separable $\operatorname{ANR}(\mathfrak{M})$-space (see the proof of VI. 3.1 in [16]) and is a $G_{8}$-subset of the complete space $O(T, Z)$.

This easily implies (c.f. examples on p. 168 of [10]).

(3) Cf. Theorem 5 of [15]. The assumptions of the last theorem are satisfied since, by $3.2,1.1$ and the theorem of Dugundji [8, p. 188], we have $E \cong E \times E^{\infty}$ if $E$ is a Hilbert space and $E \cong E \times \sum E$ otherwise. 
47. Coroltaru. Let $X, X_{1}, \ldots, X_{n}$ be as in 4.6, let $T$ be a compact finite-dimensional manifold and let $T_{1}, \ldots, T_{n} \subset T$ be disjoint closed sets. Then $\left\{f \in C(T, X): f\right.$ is non-constant and $f\left(T_{i}\right) \subset X_{i}$ for $\left.i \in[1, n]\right\}$ forms an $l_{0}$-manifold. In particular, the space of non-constant paths from $X_{1}$ to $X_{2}$ and the space of non-constant closed curves starting from $X_{1}$ form $l_{2}$-manifolds ( $\left.{ }^{4}\right)$.

The second application is:

4.8. COROLLARY. Let $X$ be a separable metric space which admits a nontrivial flow, let $A$ and $B$ be subsets of $X$, and denote by $\operatorname{Auth}_{B}(X, A)$ the space of all homeomorphism $f: X \stackrel{\text { onto }}{\longrightarrow} X$ with $f(A)=A$ and $f \mid B=i$ dentity $\left(\operatorname{Auth}_{B}(X, A)\right.$ is regarded under the compact-open topology). If $\operatorname{Auth}_{B}(X, A)$ is an $\operatorname{ANR}(\mathfrak{M})$, then it is an $l_{2}$-manifold.

Proof. Apply Proposition 4.5 and a result of Keesling [17], p. 6.

It was shown by R. Luke and W. K. Mason [21], [22], that the space $H(X)=\operatorname{Auth}_{\partial X}(X, X)$ is an $\operatorname{ANR}(\mathfrak{M})$ for every compact $[0,1]^{2}$-manifold $X$. This implies:

4.9. COROLIARX. If $X$ is a compact 2-dimensional manifold, then $H(X)$ is an $l_{2}$-manifold.

5. Products and factors of metric simplicial complexes. By a metric simplicial complex we mean here the geometric realization $X$ of a simplicial complex (say, $K$ ), endowed with the metric topology of $X$ ([16], p. 99). We shall say that the metric simplicial complex $X$ is induced by $K$ and, since only simplicial complexes are considered here, we shall sometimes write "metric complex" instead of "metric simplicial complex".

The following is an extension of a result of J. E. West [32].

5.1. Propostrion. If $X$ is a metric simplicial complex and $A$ is a set of cardinality dens $(X)$, then $X \times l_{2}^{\dagger}(A)$ is an $l_{2}^{\dagger}(A)$-manifold and $X \times$ $\times l_{2}(A) \times \sum R$ is an $l_{2}(A) \times \sum R$-manifold.

Proof. By its definition, $X$ is a closed subset of $l_{2}^{f}(A)$ and it is well known that $X \in \operatorname{ANR}(\mathfrak{M})\left([16]\right.$, p. 106). Thus $X \in \mathfrak{M}_{3} \subset \mathfrak{M}_{2}$ and the assertion follows from Theorem 4.4 .

We note that the density character of the metric complex induced by a connected simplicial complex $K$ is equal to the supremum of the cardinalities of simplices belonging to st $(v)$, the supremum being talken over all vertices $v$ of $K$.

(4) The assertion is also true if we omit the words "non-constant". This results from the following fact, which can be established by using 4.6 and a technique of Cutler: If $X \in \operatorname{ANR}(\mathfrak{M})$ and there is a $Z$-set $A$ in $X$ (see Section 6 for the definition) such that $X \backslash A$ is $a l_{2}$-manifold, then $X$ itself is an $l_{2}$-manifold. A proof will appear in author's note "Concerning $Z$-sets in ANR's and characterization of $l_{2}$-manifolds".
5.2. Proposition. Let $X$ be a metric simplicial complex and let $A$ be a set of cardinality dens $(X)$. If $X$ is complete-metrizable, then $X \times l_{2}(A)$ is an $l_{2}(A)$ - manifold.

Proof. Apply Theorem 4.5.

It is clear that a metric complex induced by a simplicial complex $K$ is complete-metrizable iff for every vertex $v$ of $K$ there is no infinite, strictly increasing sequence of simplices belonging to st $(v)([16], \mathrm{p} .107)$; in particular, every locally finite-dimensional metric complex is completemetrizable. (For locally finite-dimensional complexes the assertion of Proposition. 5.2 follows from more general results of J. E. West [31].)

Theorem 4.5 allows us also to characterize the spaces which stabilize to metric complexes (see [14] for results on spaces stabilizing to countable $C W$-simplicial complexes).

5.3. Proposition. Let $X$ be a connected metrio space and let $A$ be a set of cardinality dens $(X)$. Then the following conditions are equivalent

(a) $X$ is homeomorphic to a retract of a metric simplicial complex;

(b) $X \in \operatorname{ANR}(\mathfrak{M})$ and $X$ is a countable union of locally compact, locally finite-dimensional sets;

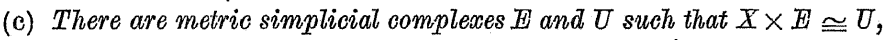
$E \cong l_{2}^{\dagger}(A)$ and $U$ is homeomorphic to an open subset of $l_{2}^{\dagger}(A)$.

Proof. Implication $(a) \Rightarrow$ (b) follows from [16], p. 106, and from the fact that for every metric simplicial complex the differences between its successive finite-dimensional skeletons are locally compact and finitedimensional.

(b) $\Rightarrow$ (c). By Theorem 4.5 the product $X \times l_{2}^{f}(A)$ is homeomorphic to an open subset of $l_{2}^{f}(A)$. Since the space $l_{2}^{f}(A)$ is homeomorphic to a metric simplicial complex [15], and since every open subset of a metric complex is homeomorphic to another metric complex, the implication is proved.

Implication $(\mathrm{c}) \Rightarrow(\mathrm{a})$ is trivial.

6. Tubular neighbourhoods with infinite-dimensional fibre. Let $h: X \rightarrow \bar{Y}$ be an embedding. By a trivial tubular neighbourhood of $h$ we mean a triple $(E, \bar{h}, U)$, where $E$ is a linear metric space, $U$ is an open subset of $Y$, and $\bar{h}: X \times E \stackrel{\text { onto }}{\rightarrow} U$ is a homeomorphism such that $\bar{h}(x, 0)=h(x)$ for all $x \in X$. The space $E$ will be called the fibre of the tubular neighbourhood, and, allowing a lack of precision, we shall sometimes say that $h$ or $U$ itself form a tubular neighbourhood of $h$. If $X$ is a subset of $Y$ and $i$ denotes the inclusion, then we shall say "tubular neighbourhood of $X$ " instead of "tubular neighbourhood of $i$ ". It is easy to see that an open set $U \subset Y$ is a trivial tubular neighbourhood of an embedding $h: X \rightarrow Y$ if and only if it is a trivial tubular neighbourhood of the set $h(X)$. 
Let $E$ be a normed linear space. An embedding $h: X \rightarrow Y$ is said to be $E$-deficient if $h$ is a closed map and there is a homeomorphism $f: Y \stackrel{\text { onto }}{\rightarrow} Y \times \mathbb{E}$ such that $f h(X) \subset Y \times\{0\}$. Obviously, the space $X$ admits

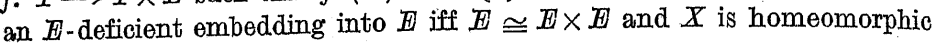
to a closed subset of $E$.

6.1. LemMa. Let $E$ be a locally convex linear metric space such that $\mathbb{E} \cong \mathbb{E} \times[0,1)$. If $X$ is a space satisfying $O X \times \mathbb{E} \cong \mathbb{E}$, then every $\mathbb{E}$-de-

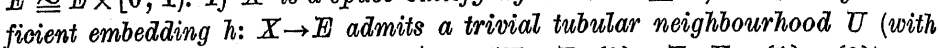
fibre $\mathbb{E})$ such that $(\mathbb{E}, \mathbb{E} \backslash U, h(X)) \cong(C X \times \mathbb{E},\{0\} \times \mathbb{Z}, X \times\{1\} \times\{0\})$.

If the assumption $O X \times \mathbb{E} \cong \mathbb{E}$ is replaced by $X \times \mathbb{E} \cong \mathbb{H}$, then $\mathbb{E}$ itself is a tubular neighbourhood with fibre $\mathbb{E}$ of every $\mathbb{E}$-deficient embedding of $X$ into $E$.

Proof. Let $E_{1}=C X \times E, A_{1}=X \times\{1\} \times\{0\}$ and $A=h(X)$. The embedding $h$ induces in a natural way the homeomorphism $g: A_{1} \stackrel{\text { onto }}{\longrightarrow} A$. Then, $E_{1}$ is homeomorphic to $E$ and the sets $A_{1}$ and $A$ are $E$-deficient in $E_{1}$ and $E$ respectively (observe that $E \cong E \times \mathbb{E}$ ); thus by a lemma of $\nabla$. L. Klee ([19], p. 36) there is a homeomorphism $\bar{g}: \mathbb{H}_{\mathbf{1}} \stackrel{\text { onto }}{\longrightarrow} \mathbb{E}$ such that $\bar{g} \mid \mathcal{A}_{1}=g$. We set $U=\bar{g}(X \times(0,1] \times E)$; it is clear that $U$ is a trivial tubular neighbourhood of $h$ with fibre $E \times(0,1] \cong E$. The proof of the second assertion is left to the reader.

Let $I$ denote the unit interval $[0,1]$. A subset $X$ of a topological space $E$ is said to be a $Z$-set in $E$ iff $X$ is closed in $Z$ and, for every $n$, the set $\left\{f \in C\left(I^{n}, E\right): f\left(I^{n}\right) \cap X=\emptyset\right\}$ is dense in $O\left(I^{n}, X\right)\left({ }^{5}\right)$. An embedding $h: X \rightarrow Z$ will be called a $Z$-embedding iff $h(X)$ is a $Z$-set in $E$.

We shall use the following lemma:

6.2. Lemma. Let $E$ be a locally convex linear metric space such that $E \cong E^{\infty}$ or $E \cong \sum E$. Then

(a) $E \cong E \times[0,1)$

(b) An embedding into a paracompact $E$-manifold is $E$-deficient if and only if it is a $Z$-embedding.

For the proof see respectively [5], p. 184 (observe that $T$ has either $R^{\infty}$ or $\sum R$ as factor) and [27], §6.

Combining 6.2, 3.2 and 6.1 we get (cf. the footnote $\left({ }^{8}\right)$ ):

6.3. THEOREM. Let $E$ be a space of the form $\mathbb{E}_{i}(A)$, where $i \in[1,4]$ and $A$ is an infinite set, and let $h$ be a $Z$-embedding into $Z$ of a space

(5) This definition differs from R. D. Anderson's [1] original one; however, if $X$ has a base consisting of homotopy trivial sets, then the $Z$-sets as defined above coincide with the "sets with Property $Z$ " of [1] and also with the sets which are homotopy negligible (in sense of [9]) in "every open subset of $X$.
$X \in \operatorname{ANR}(\mathfrak{M})$. Then there is a trivial tubular neighbourhood $U$ of $h$ such that $(E, \mathbb{E} \backslash U, h(X)) \cong(O X \times E,\{0\} \times E, X \times\{1\} \times\{0\})$. If, in addition, $X$ is contractible (i.e. $X \in \mathrm{AR}(\mathfrak{M}))$, then $E$ itself is a tubular neighbourhood of every $Z$-embedding $h: X \rightarrow E$.

Let us also note that, conversely, if a closed embedding $h: X \rightarrow Y$ $\epsilon \operatorname{ANR}(\mathfrak{M})$ admits a tubular neighbourhood with infinite-dimensional fibre, then $X$ must be an $\operatorname{ANR}(\mathfrak{M})$ and $h$ must be a $Z$-embedding. (A proof easily follows from the results of Eells and Kuiper [9]).

6.4. CoRollary. Let $E$ be as in Theorem 6.3 , let $M$ be a paracompact manifold modelled on $\mathbb{E}$ and let $h: X \rightarrow M$ be a $Z$-embedding. If $X \in \mathrm{ANR}(\mathfrak{M})$, then $h$ admits a trivial tubular neighbourhood with fibre $E$.

Proof. Without loss of generality one can assume that $M$ is a connected open subset of $\mathbb{E}$ (see 4.4). By Lemma $6.2(\mathrm{~b})$ there is a homeomorphism $g: M \stackrel{\text { onto }}{\longrightarrow} M \times E \times R$ such that $g h(X) \subset M \times\{0\} \times\{0\}$. Set

$$
g_{1}(m, t, \lambda)=(m, t, \lambda+1 / \alpha(m)), \quad(m, t, \lambda) \in M \times \mathbb{E} \times R,
$$

where $\alpha: E \rightarrow[0,1]$ is a continuous function with $\alpha^{-1}(0)=E \backslash M$. Then $g_{1} g$ is a homeomorphism of $M$ onto $M \times E \times R$ and $g_{1} g h$ is an $E$-deficient embedding of $X$ into $Z \times E \times R \cong E$. By 6.3 there is a tubular neighbourhood $U_{1}$ (with fibre $E$ ) of $g_{1} g h$ in $E \times E \times R$, and the standard arguments show that we can assume $U_{1}$ to be contained in the open neighbourhood $M \times \mathbb{E} \times R$ of $g_{1} g h(X) . g^{-1}\left(U_{1}\right)$ is the required tubular neighbourhood of $\dot{h}$ in $M$.

\section{We note also}

6.5. COROLLARY. Let $\mathbb{E}$ be a linear metric space which has $l_{2}$ as factor (e.g. let $E$ be any infinite-dimensional Fréchet space). Then, every compact ANR-subset of $E$ admits a trivial tubular neighbourhood $U$ with fibre $E$ such that $(E, E \backslash U, X) \cong(C X \times E,\{0\} \times E, X \times\{1\} \times\{0\})$; moreover, $E$ is a trivial tubular neighbourhood with fibre $\mathbb{B}$ of each of its compact $\mathrm{AR}$ subsets.

The proof is the same as that of Lemman 6.1, by using an easy generalization of Klee's homogeneity theorem (cf. [32], p. 262).

Finally we have

6.6. THEOREM. Let $X$ be an $\operatorname{ANR}(\mathfrak{M})$. Then there is a normed linear space $\mathbb{Z}$ such that: (1) dens $(E) \cong$ dens $(X),(2) X$ admits $E$-deficient em-

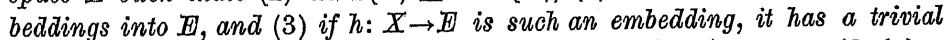

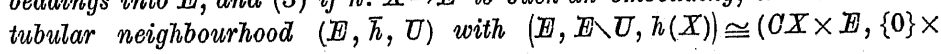
$\times \mathbb{E}, X \times\{1\} \times\{0\})$.

Proof. By Theorem 3.1 there is a normed linear space $F$ such that $\operatorname{dens}(F)=\operatorname{dens}(X)$ and $O X \times F^{\prime} \cong F$ (cf. the proof of Theorem 4.2). We let $E=\sum_{l_{1}} F$. Then $B \cong E \times E$ and $O X$ admits a closed embedding into $E$; 5 - Fundamenta Mathematicae, T. LXXXVI 


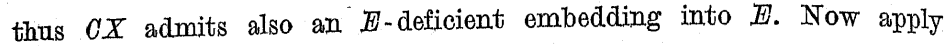
Lemma 6.1.

Let us note that if $(E, \bar{i}, U)$ is a trivial tubulair neighbourhood of a set $X \subset Y$, then there is a (straight deformation) retraction of $U$ onto $X$

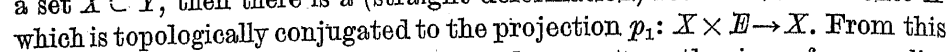
point of view, Theorem 6.6 can be read as a strengthening of an earlier result of D. W. Henderson ([13], p. 748).

Addendum. The constructions of 2.1 admit easy geometric interpretation, i.e. the proof of 2.1 leads to an $F$-normed linear space $(H,||||||)$ and to a closed embedding $h: X \rightarrow H$ such that the metric

$$
\varrho\left(x_{1}, x_{2}\right)=\|\| h\left(x_{1}\right)-h\left(x_{2}\right)\|\|
$$

satisfies the assertion of 2.1 ; moreover, the space $H$ can be built in such a way that $H \cong E^{\infty}$. This implies that if $X$ is a retract of a locally convex linear metric space $E$, then $X \times \sum E \cong \sum E$, and if additionally $X$ is complete-metrizable, then $X \times E^{\infty} \cong \mathbb{E}^{\infty}$. As a corollary one gets that for any Fréchet space $F, F^{\infty}$ is homeomorphic to a Hilbert space. Details will appear in [29].

\section{References}

[1] R. D. Anderson, On topological infinite deficiency, Mich. Math. J. 14 (1967), pp. 265-283.

[2] - and R. M. Schori, Factors of infinite-dimensional manifolds, Trans. Amer. Math. Soc. 142 (1969), pp. 315-330.

[3] R. Arens and J. Eells, On embedding uniform and topological spaces, Pacific J. Math. 6 (1956), pp. 397-403.

[4] C. Bessaga and A. Pełczyński, Some remarks on homeomorphisms of $F$-spaces, Bull. Acad. Polon. Sci. Sér. Sci. Math. Astronom. Phys. 10 (1962), pp. 265-270.

[5] - The estimated extension theorem, homogeneous collections and skeletons, and their applications to topological classification of linear metric spaces and convex sets, Fund. Math. 69 (1970), pp. 153-190.

[6] - On spaces of measurable functions, Studia Math. 44 (1972), pp. 597-615.

[7] K. Borsuk, Theory of Retracts, Warszawa 1967.

[8] J. Dugundji, Topology, Boston 1966.

[9] J. Eells and W. H. Kuiper, Homotopy negligible subsets of infinite-dimensional manifolds, Compositio Math. 21 (1969), pp. 155-261.

[10] R. Geoghegan, On spaces of homeomorphisms, embeddings and functions $I$, Topology 11 (1972), pp. 159-177.

[11] D. W. Henderson, Micro-bundles with infinite-dimensional fibres are trivial, Inventiones Math. 11 (1970), pp. 293-303.

[12] - Oorrections and extensions of two papers about infinite-dimensional manifolds, Gen. Topology and Appl. 1 (1971), pp. 321-327.

[13] - Applications of infinite-dimensional manifolds to quotient spaces of complete ANR's, Bull. Acad. Polon. Sci. Sér. Sci. Math. Astronom. Phys. 19 (1971), pp. 747-753.
[14] D. W. Henderson, A simplicial complex whose products with any finitedimensional absolute retract is a simplicial complex, Gen. Topology and Appl. 3 (1973), pp. 81-83.

[15] - and J. E. West, Triangulated infinite-dimensional manifolds, Bull. Amer. Math. Soc. 76 (1970), pp. 655-660.

[16] S. T. Hu, Theory of Retracts, Detroit 1965.

[17] J. Keesling, Using flows to construct Hilbert space factors of function spaces, Trans. Amer. Math. Soc. 161 (1971), pp. 1-24.

[18] V. J. Klee, On the Borelian and projective types of linear subspaces, Math. Scand. 6 (1958), pp. 189-199.

[19] - Some topological properties of convex sets, Trans. Amer. Math. Soc. 78 (1955), pp. $30-45$.

[20] - MIappings into normed linear spaces, Fund. Math. 49 (1960), pp. 25-34.

[21] R. Luke and W. K. Mason, The space of homeomorphisms on a compact twomanifold is an absolute neighbourhood retract, Trans. Amer. Math. Soc. 164 (1972), pp. 275-285.

[22] W. K. Mason, The space of all self-homeomorphisms of a two-cell which fix the cell's boundary is an absolute retract, ibidem 161 (1971), pp. 185-205.

[23] E. Michael, Local properties of topological spaces, Duke Math. J. 21 (1954), pp. 163-171.

[24] - A short proof of the Arens-Eells embedding theorem, Proc. Amer. Math. Soc. 15 (1964), pp. 415-416.

[25] R. Palais, Homotopy theory of infinite-dimensional manifolds, Topology 5 (1966), pp. 1-16.

[26] R. Schori, Topological stability for infinite-dimensional manifolds, Compositio Math. 23 (1971), pp. 87-100.

[27] H. Torunczyk, $(G, K)$-absorbing and skeletonized sets in metric spaces, Dissertationes Math. (to appear).

[28] - Oompact absolute retracts as factors of the Hilbert space, Fund. Math. 83 (1973), pp. 75-84.

[29] - On Cartesian fastors and the topological olassification of linear metric spaces, Fund. Math. (to appear).

[30] - A short proof of Hausdorff theorem on extending metrics, Fund. Math. 77 (1972), pp. 190-193.

[31] J. E. West, Products of complexes and F'réchet spaces which are manifolds, Trans, Amer. Math. Soc. 166 (1972), pp. 317-337.

[32] - The ambient homeomorphy of an incomplete subspace of infinite-dimensional Hilbert space, Pacific J. Math. 34 (1970), pp. 257-267.

Regu par la Rédaction le 12. 3. 1973 